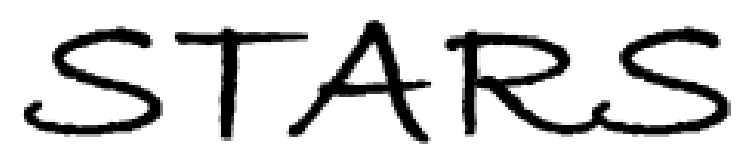

University of Central Florida

STARS

$1-1-2010$

\title{
Spectral and temperature dependence of two-photon and free- carrier absorption in InSb
}

\author{
Peter D. Olszak \\ University of Central Florida \\ Claudiu M. Cirloganu \\ University of Central Florida \\ Scott Webster \\ University of Central Florida \\ Lazaro A. Padilha \\ University of Central Florida \\ Shekhar Guha
}

Find similar works at: https://stars.library.ucf.edu/facultybib2010

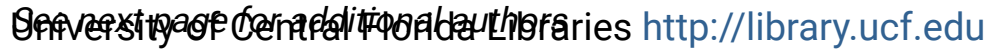

This Article is brought to you for free and open access by the Faculty Bibliography at STARS. It has been accepted for inclusion in Faculty Bibliography 2010 s by an authorized administrator of STARS. For more information, please contactSTARS@ucf.edu.

\section{Recommended Citation}

Olszak, Peter D.; Cirloganu, Claudiu M.; Webster, Scott; Padilha, Lazaro A.; Guha, Shekhar; Gonzalez, Leonel P.; Krishnamurthy, Srini; Hagan, David J.; and Van Stryland, Eric W., "Spectral and temperature dependence of two-photon and free-carrier absorption in InSb" (2010). Faculty Bibliography 2010s. 609. https://stars.library.ucf.edu/facultybib2010/609

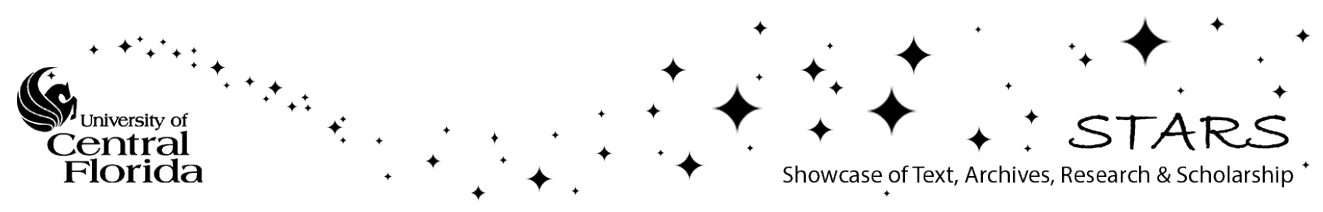


Authors

Peter D. Olszak, Claudiu M. Cirloganu, Scott Webster, Lazaro A. Padilha, Shekhar Guha, Leonel P. Gonzalez, Srini Krishnamurthy, David J. Hagan, and Eric W. Van Stryland 


\title{
Spectral and temperature dependence of two-photon and free-carrier absorption in InSb
}

\author{
Peter D. Olszak, ${ }^{1}$ Claudiu M. Cirloganu, ${ }^{1}$ Scott Webster, ${ }^{1}$ Lazaro A. Padilha, ${ }^{1}$ Shekhar Guha, ${ }^{2}$ Leonel P. Gonzalez, ${ }^{2}$ \\ Srini Krishnamurthy, ${ }^{3}$ David J. Hagan, ${ }^{1}$ and Eric W. Van Stryland ${ }^{1, *}$ \\ ${ }^{1}$ CREOL \& FPCE, The College of Optics and Photonics, University of Central Florida, 4000 Central Florida Boulevard, \\ Orlando, Florida 32826, USA \\ ${ }^{2}$ Air Force Research Laboratory, Materials and Manufacturing Directorate, Wright Patterson Air Force Base, Ohio 45433, USA \\ ${ }^{3}$ SRI International, 333 Ravenswood Avenue, Menlo Park, California 94025, USA
}

(Received 10 June 2010; revised manuscript received 6 October 2010; published 13 December 2010)

\begin{abstract}
The nonlinear absorption spectrum of InSb was measured using a combination of tunable $\sim 160 \mathrm{fs}, \sim 10 \mathrm{ps}$, and $\sim 150 \mathrm{~ns}$ IR sources along with a cryostat for controlling the sample temperature to vary the band gap energy from 0.17 to $0.23 \mathrm{eV}$. The measured nonlinear optical properties in InSb are consistent with those predicted by the models which include two- and three-photon absorption (2PA and 3PA), multiphoton generated free-carrier absorption (FCA) and various recombination mechanisms. Temperature-dependent Z-scan and nonlinear transmission measurements yield information on the temperature and spectral dependence of 2PA, FCA, and carrier recombination processes of Shockley-Read-Hall, and Auger mechanisms. We find good agreement between the measured and the modeled nonlinear properties is possible only when the recently predicted temperature dependence of the FCA is considered. The wavelength- and temperature-dependent 2PA and 3PA coefficients in InSb were experimentally obtained. The inferred values of the 2PA and 3PA are consistent with the scaling rules of a simple two-parabolic band model. We further determine recombination rates from nonlinear transmittance of nanosecond pulses of $\mathrm{CO}_{2}$ laser.
\end{abstract}

DOI: 10.1103/PhysRevB.82.235207

PACS number(s): 42.70.Nq, 42.70.Mp, 78.40.Fy, 71.28.+d

\section{INTRODUCTION}

Multiphoton absorption has been a topic of research for 80 years, starting with the theory for two-photon absorption (2PA) developed by Maria Goeppert-Mayer ${ }^{1}$ in the early 1930s but experiments did not begin until 1961 (Ref. 2) just after the invention of lasers. Generalized theories for multiphoton absorption in semiconductors were developed over the following decades. ${ }^{3-6}$ Keldysh used tunneling theory to determine the multiphoton transition rate. ${ }^{3}$ Then, Jones and Reiss used both the tunneling approach (with Volkov-type "dressed" wave functions) and perturbation theory assuming circular polarization, showing that the results are identical for the calculated transition rates. ${ }^{4}$ The theory for multiphoton interband absorption in semiconductors, assuming linear polarized radiation and two parabolic bands, produced simple scaling rules for $\mathrm{N}$-photon absorption, as done by Brandi and de Araujo ${ }^{5}$ using Keldysh's approach in 1983 and by Wherrett ${ }^{6}$ in 1984 using perturbation theory. Both theories predict the absorption coefficient to be proportional to the band gap energy as $E_{g}^{-(4 N-5)}$, leading to $E_{g}^{-3}$ for $2 \mathrm{PA}$ and $E_{g}^{-7}$ for three-photon absorption (3PA). These theories give identical results for 2PA, however, they are not expected to give exact quantitative agreement with experiment and are typically scaled by an empirical constant when being compared to data. ${ }^{7,8}$

2PA in semiconductors was experimentally shown in Ref. 11 to scale with $E_{g}^{-3}$ as predicted, thus demonstrating the utility of the simple two-parabolic band approach as used by Wherrett and de Araujo. ${ }^{5,6}$ The 2PA coefficient in semiconductors, $\alpha_{2}$, can be estimated by,

$$
\alpha_{2}=K_{2} \frac{\sqrt{E_{p}}}{n^{2} E_{g}^{3}}\left[\frac{\left(2 \hbar \omega / E_{g}-1\right)^{3 / 2}}{\left(2 \hbar \omega / E_{g}\right)^{5}}\right]=K_{2} \frac{\sqrt{E_{p}}}{n^{2} E_{g}^{3}} F_{2},
$$

where $n$ is the refractive index, $E_{p}$ is the Kane momentum parameter, ${ }^{9} \hbar \omega$ is the photon energy, and $F_{2}$ defines the spectral dependence. ${ }^{10}$ The predicted $2 \mathrm{PA}$ value is then calculated using a value for $K_{2}=1.94 \mathrm{eV}^{5 / 2} \mathrm{~cm} / \mathrm{MW}$ calculated from first principles and universal constants or with $K_{2}=3.10 \mathrm{eV}^{5 / 2} \mathrm{~cm} / \mathrm{MW}$ obtained empirically by fitting the 2PA measured using many semiconductors with different band gap energies. ${ }^{11,12}$ This empirical constant used in the scaling theory allowed prediction of $\alpha_{2}$ in other materials. For example, Ref. 11 employs the scaling function for $\mathrm{InSb}$ using $E_{g}=0.18 \mathrm{eV}$ at $10.6 \mu \mathrm{m}$ and predicts $\alpha_{2}=6.8 \mathrm{~cm} / \mathrm{MW}$. Experimentally reported values for the 2PA coefficient of InSb at room temperature have ranged from 0.2 to $20 \mathrm{~cm} / \mathrm{MW} .{ }^{13}$ This inconsistency is due to significant effects from free-carrier absorption (FCA) and freecarrier refraction (FCR) in InSb. ${ }^{14}$ Other difficulties associated with these measurements in the midinfrared wavelength regime include the lack of detectors with high sensitivities and low noise, alignment issues, as well as beam quality and pulse shape.

In this paper, we present an investigation of both the temperature and spectral behavior of 2PA and FCA in InSb along with the relevant carrier dynamics in the $8-12 \mu \mathrm{m}$ wavelength band. Three antireflection coated undoped samples are used with similar thicknesses: $0.54 \mathrm{~mm}$ for femtosecond Z-scans (see Refs. 15 and 16) and picosecond pump probe, $0.65 \mathrm{~mm}$ for Fourier-transform infrared (FTIR) spectroscopy and picosecond Z-scan, and $0.55 \mathrm{~mm}$ for nanosecond experiments up to the damage threshold. Semiconductor band gap energies are known to vary with temperature, ${ }^{17}$ therefore $\alpha_{2}$ in InSb is expected to vary accordingly, i.e., with $E_{g}^{-3}$. While the FCA cross section has been generally accepted in the past to be a constant for the temperatures and wavelengths used in this study, ${ }^{18}$ it has been recently predicted that it varies significantly with temperature as well as wavelength. ${ }^{19} \mathrm{We}$ 
employ a wide range of pulsewidths to measure the nonlinear absorption and account for the increasing importance of freecarrier effects for longer pulses in the determination of nonlinear absorption coefficients; justification for this will be given in Sec. II. The methodology is to study the ultrafast multiphoton absorption spectrum using short pulses and then free-carrier effects using progressively longer pulses. The complex effects of free carriers on the dynamics when using the longest pulses are accounted for by adding more parameters to the model as needed.

Linear and nonlinear transmission measurements are used to characterize the temperature and spectral behavior of FCA. We make use of the known temperature dependence of the band gap and to verify $2 \mathrm{PA}$ scaling rules we use a tunable picosecond laser system and a nitrogen cryostat to control the sample temperature. Values of 2PA coefficients obtained at room temperature using a tunable femtosecond laser system are also presented along with an investigation of nonlinear absorption and free-carrier recombination using a nanosecond $\mathrm{CO}_{2}$ gas laser system and a helium cryostat to control the sample temperature. Great care was taken to ensure that all of the transmitted light was collected, as this may be a significant source of error due to the large FCR and other nonlinear index of refractions. ${ }^{14}$ Data obtained over this large temporal pulse range and in the spectral range measured are self-consistent and agree with the models presented below.

\section{MODELING PARAMETERS}

The modeling of our data utilizes a propagation equation for the irradiance $I$, coupled to a rate equation for the freecarrier population $N$, as shown below

$$
\begin{aligned}
& \frac{d I}{d z}=-\alpha_{1} I-\alpha_{2} I^{2}-\alpha_{3} I^{3}-\sigma_{F C A} N_{p g} I, \\
& \frac{d N_{p g}}{d t}=\frac{\alpha_{2} I^{2}}{2 \hbar \omega}+\frac{\alpha_{3} I^{3}}{3 \hbar \omega}-A\left(N-N_{0}\right) \\
&-B\left(N^{2}-N_{0}^{2}\right)-C N\left(N^{2}-N_{0}^{2}\right) \\
&=\frac{\alpha_{2} I^{2}}{2 \hbar \omega}+\frac{\alpha_{3} I^{3}}{3 \hbar \omega}-A N_{p g}-B N_{p g}\left(N_{p g}+2 N_{0}\right) \\
&-C N_{p g}\left(N_{p g}+N_{0}\right)\left(N_{p g}+2 N_{0}\right) .
\end{aligned}
$$

Here $\alpha_{1}$ is the one-photon absorption (1PA), $\alpha_{2}$ the 2PA, and $\alpha_{3}$ the 3PA coefficient. The total free-carrier population $N$ is the sum of initial or thermal carriers, $N_{0}$, and photogenerated carriers, $N_{p g}$. The FCA cross section, $\sigma_{\mathrm{FCA}}$, is the sum of the cross section for electrons and holes. The cross section for holes is about 40 times larger than that for electrons due to the direct (nonphonon-assisted) heavy-hole to light-hole transition, so FCA is dominated by free-hole absorption. For the wavelengths used, 1PA across the band gap is not energetically possible. Linear absorption from defects and impurities can be included in $\alpha_{1}$; however, our FTIR results fit well without including such linear losses in our undoped samples with a defect concentration of $\sim 9 \times 10^{13} \mathrm{~cm}^{-3}$. The Shockley-Read-Hall (SRH) recombination rate $A$, spontane- ous radiative recombination rate $B$, and Auger recombination (AR) rate $C$ are neglected for subnanosecond measurements while FCA and recombination are neglected for subpicosecond measurements as will be discussed later in this manuscript. We note that the physical processes that dominate in the spectral region of these experiments become quite different when photon energies either above or much further below the band gap are employed..$^{20-22}$

The advantage of using a range of pulsewidths to study the nonlinear optical properties of InSb is that we can test the model through various simplifying assumptions for the various pulsewidths from simply 2PA for femtosecond pulses to increasing complexity as the pulsewidth increases. For example, instantaneous absorption processes such as multiphoton absorption are more easily investigated using ultrashort pulsewidths so that large irradiances can be obtained with negligible effects from photogenerated carriers which build up during the pulse. The picosecond range is ideal for studying nonlinear absorption including multiphoton absorption and population dependent FCA since recombination effects are negligible. For nanosecond pulsewidths, nonlinear absorption and carrier recombination processes yield effective lifetimes in the nanosecond range and must be considered, further complicating the analysis. Thus, the methodology is to study the material parameters with progressively longer pulses adding more parameters to the model as they become significant.

\section{EXPERIMENTAL AND DISCUSSION}

\section{A. Linear spectroscopy experiments}

Measurements of the linear transmittance from 8 to $12 \mu \mathrm{m}$ at room temperature exhibit FCA due to the thermal population in InSb. The FCA cross section can therefore be isolated through linear transmittance measurements. To determine the temperature dependence of the FCA spectrum, the linear transmittance is measured at different temperatures via FTIR using a Thermo model 100 FTIR spectrometer and a microminiature refrigerator (MMR model K-77) nitrogen cryostat to control the sample temperature. Our FCA values compared to theory are shown in Fig. 1, demonstrating that the FCA cross section is not constant, but has a spectral and temperature dependence. ${ }^{19}$

The carrier population is taken to be equal for holes and electrons. This is a valid assumption since our anti-reflection coated sample (Galaxy Compound Semiconductors Inc. $\langle 111\rangle$ cut single crystal) is undoped with a defect concentration of approximately $9 \times 10^{13} \mathrm{~cm}^{-3}$, and this is approximately equal to the thermal population at $160 \mathrm{~K}$. The thermal population at room temperature is approximately 2 $\times 10^{16} \mathrm{~cm}^{-3}$. The Boltzmann approximation for carrier concentration leads to an error of almost $10 \%$ due to the degenerate population and a more accurate calculation based on the Fermi-Dirac distribution is used. At $80 \mathrm{~K}$, the thermal density of carriers is much smaller $\left(5 \times 10^{9} \mathrm{~cm}^{-3}\right)$ while the population is nondegenerate for temperatures below $200 \mathrm{~K}$. The carrier population becomes too small at low temperatures making it difficult to measure the loss with this experiment, as the total linear absorption is less than $5 \%$ below 200 

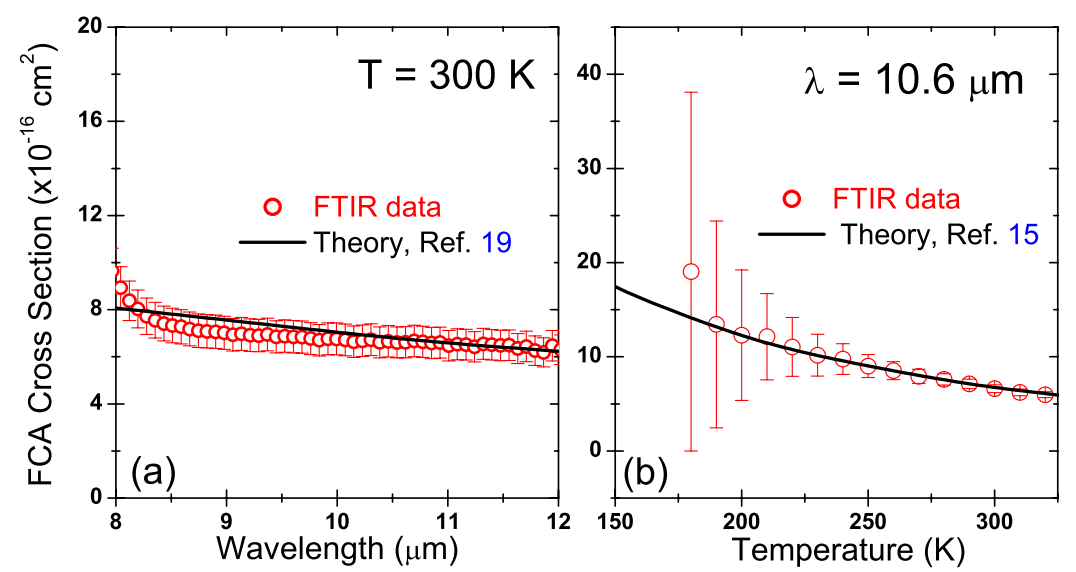

FIG. 1. (Color online) FCA spectral theory (solid lines) from Ref. 19 and data (open circles) from linear absorption measurements in a 0.65-mm-thick InSb sample at (a) $300 \mathrm{~K}$ vs wavelength and (b) $10.6 \mu \mathrm{m}$ vs temperature.
$\mathrm{K}$. This leads to large experimental uncertainties below 200 $\mathrm{K}$ so that the total experimental error (plus or minus one standard deviation giving $68 \%$ confidence for a normal distribution) for FCA is estimated to be $\pm 10 \%$ at $300 \mathrm{~K}$ and $\pm 100 \%$ at $180 \mathrm{~K}$. For the low-temperature range, we use the FCA cross sections determined from fitting the picosecond Z-scans (to be discussed in the following sections), which agrees with the theoretical values reported in Ref. 19.

Although the FCA cross section in InSb was previously accepted to be a constant, ${ }^{18,23}$ for the range of temperatures and wavelengths used in our experiments, we have found from FTIR measurements of the linear absorption and picosecond Z-scan measurements (see the following sections) that it is actually temperature dependent. Moreover, it is in agreement with the recent predictions of Ref. 19 as shown in Fig. 1. The FCA is temperature dependent due to the change in the Fermi-Dirac distribution of carriers. This distribution is narrower for lower temperatures and a larger fraction of holes are available for absorbing longer wavelength photons due to the constraints for energy and momentum conservation. This results in a larger FCA cross section and a redshifted absorption edge for low temperature. ${ }^{19}$ The upswing of the data near $8 \mu \mathrm{m}$ in Fig. 1(a) might be due to some unaccounted reflection loss since the broadband AR coating was optimized for $10 \mu \mathrm{m}$; however, the band edge is near $7 \mu \mathrm{m}$ and the linear loss starts before the band edge due to states near the edge.

\section{B. Femtosecond nonlinear experiments}

The absorptive bound electronic effects can be isolated from FCA by measuring the nonlinear transmittance (in the
2PA or 3PA wavelength range respectively) using focused femtosecond pulses to achieve large irradiance with small energies. The 2PA spectrum of a $0.54 \mathrm{~mm}$ thick intrinsic antireflective coated InSb sample at room temperature is measured by the Z-scan technique ${ }^{15,16}$ using a regeneratively amplified femtosecond (Clark-MXR, model 2010) Ti:Sapphire laser operating at $1 \mathrm{kHz}(\sim 2 \mathrm{~mJ}$ per pulse $)$ at $775 \mathrm{~nm}$, which pumps a (Light Conversion Ltd., model TOPAS) optical parametric generator/amplifier and difference frequency generator (OPG/OPA/DFG). Examples of data and fitting are shown in Fig. 2. A beam waist $w_{0}$, defined as half width at $1 / e^{2}$ of the maximum $\left(\mathrm{HW} / e^{2} M\right)$, of 40 to $60 \mu \mathrm{m}$ (depending on wavelength) is determined from fitting the $\mathrm{InSb}$ $\mathrm{Z}$-scan results across the measured spectral range from 8 to $12 \mu \mathrm{m}$.

For Z-scan data that results from pure 2PA, the full width at half maximum (FWHM) of the open-aperture Z-scan curve is equal to twice the Rayleigh range and the beam waist is determined with an error (68\% confidence) of $\sim \pm 5 \%$. In the cases where only $2 \mathrm{PA}$ is present this is as accurate as using a knife edge scan or pinhole scan. Although these experiments have the advantage of independently measuring multiphoton absorption, the small energies used make it difficult to align the setup and accurately calibrate the energy incident on the sample (68\% confidence errors were estimated to be around $\pm 30 \%$ with a calibration performed with a Laser Precision RK-5100 pyroelectric radiometer with a lock-in amplifier). The spectrum of the pulse was measured using a monochrometer (Oriel, Cornerstone 130 M-74000, 150 lines/mm Au coated grating) with a mercury cadmium telluride detector. Difficulties were encountered while per-
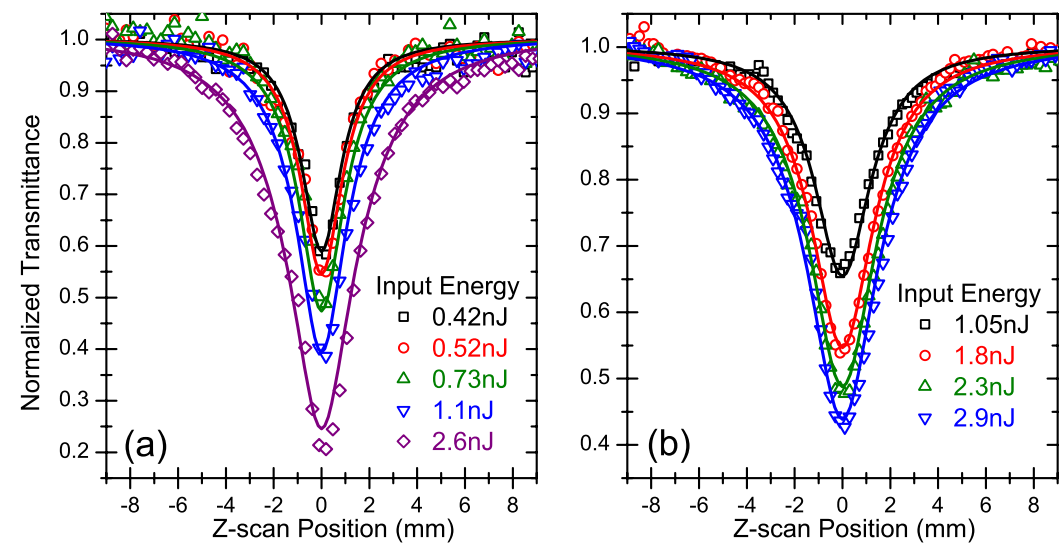

FIG. 2. (Color online) Open-aperture Z-scan data at several input pulse energies using $300 \mathrm{~K}$ a 0.54-mm-thick InSb sample at (a) $9 \mu \mathrm{m}$ fit with $\tau_{\mathrm{FWHM}}=370 \mathrm{fs}, w_{0}=45 \mu \mathrm{m}, \alpha_{2}=2.9 \mathrm{~cm} / \mathrm{MW}$ and (b) $11.5 \mu \mathrm{m}$ fit with $\tau_{\mathrm{FWHM}}=500 \mathrm{fs}$, $w_{0}=63 \mu \mathrm{m}, \alpha_{2}=2.0 \mathrm{~cm} / \mathrm{MW}$. 


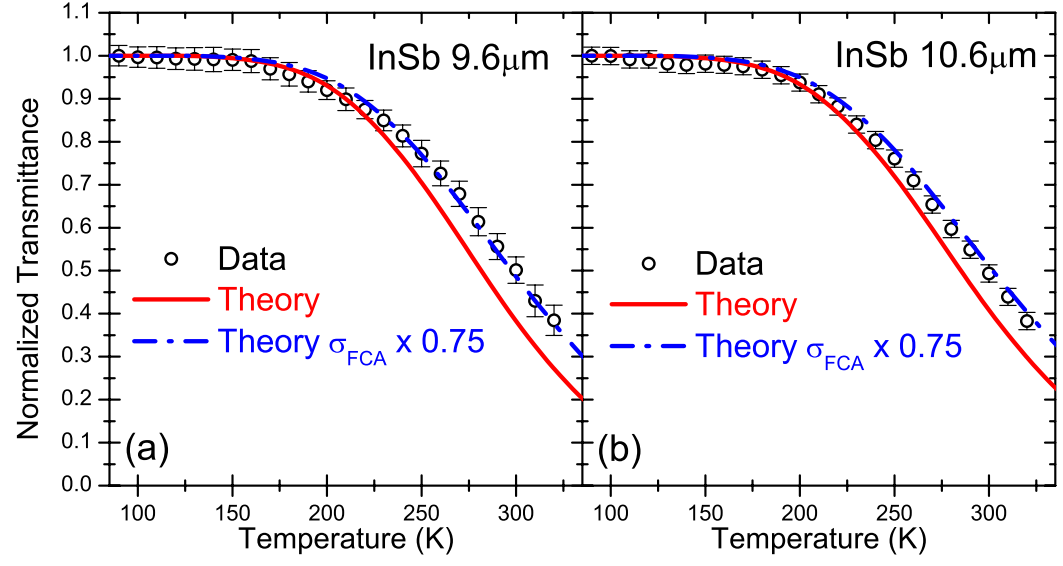

FIG. 3. (Color online) Linear transmission data in a $0.65-\mathrm{mm}$-thick InSb sample obtained at (a) $9.6 \mu \mathrm{m}$ and (b) $10.6 \mu \mathrm{m}$, using $\sim 10 \mathrm{ps}$ FWHM pulsewidths, compared to transmission using theory from Ref. 19 for FCA and the same theoretical absorption reduced by $25 \%$. forming autocorrelations on these pulses as the small energies available prevented us from obtaining reliable results. However, we measured the spectral widths for the wavelength range considered (between 8.5 and $9.5 \mu \mathrm{m}$ ). At each wavelength, based on the measured spectra, the corresponding transform limited pulsewidths was calculted to be about 160 fs FWHM. The dispersion of the ZnSe and BaF optics was then considered at each wavelength to calculate the pulsewidth at the sample where the pulse was broadened to a maximum of $\sim 500$ fs FWHM. This assumption allowed us to calculate the minimum pulsewidth possible at the sample, and implicitly, a lower limit for the 2PA coefficient. Total absolute experimental error (68\% confidence) for 2PA is estimated to be $\pm 50 \%$. Results for the $2 \mathrm{PA}$ spectrum at room temperature from femtosecond data are discussed later along with picosecond data.

\section{Picosecond nonlinear experiments}

The linear transmittance as a function of temperature is also measured at several wavelengths using the experimental picosecond Z-scan setup with small energies while the sample is kept stationary and out of focus to ensure negligible nonlinear effects. This allows the characterization of FCA dependence on temperature before any nonlinear measurements. It is found that our linear absorption measurements agree within $\pm 25 \%$ with the product of the theoretical cross section and the population density (note that both cross section and population are temperature dependent) as suggested in Ref. 19. Data at room temperature for 9.6 and $10.6 \mu \mathrm{m}$ are shown in Fig. 3. These FCA values also agree with the results from FTIR measurements. As mentioned in the previous section, at temperatures below $180 \mathrm{~K}$ where the thermal population is orders of magnitude smaller, the linear absorption is so small that the cross section cannot be determined with useful precision. To determine the FCA cross section at low temperature, we use the nonlinear absorption of photogenerated carriers and compare our experimentally obtained values to those predicted theoretically. ${ }^{19}$

The FCA due to photogenerated population $N(t)$ in Eq. (4) can be measured in combination with 2PA or 3PA by the $\mathrm{Z}$-scan technique using picosecond pulses. The more energy that is absorbed by 2PA, the more important the contribution from FCA becomes. The irradiance where the absorption contributions from 2PA and FCA are approximately equal is called the critical irradiance, $I_{c r},{ }^{24}$ and is estimated to be $6 \mathrm{MW} / \mathrm{cm}^{2}$ for a $10 \mathrm{ps}$ pulse at $10 \mu \mathrm{m}$ considering only 2PA and FCA without carrier recombination or diffusion. The total accumulated carrier population for this case is determined by the integral,

$$
N(t)=\int_{-\infty}^{t} d t^{\prime} \frac{\alpha_{2} I\left(t^{\prime}\right)^{2}}{2 \hbar \omega} .
$$

These measurements are useful for determining the shape of the 2PA and FCA spectrum at different temperatures for comparison to theory and scaling rules. Temperaturedependent picosecond Z-scan measurements of a $0.65-\mathrm{mm}$ thick intrinsic antireflection coated InSb sample are performed using a $10 \mathrm{~Hz}$ modelocked Nd:YAG laser (EKSPLA, model PL2143) pumping an OPG/OPA/DFG (model: PG401/DFG) with tunability from $420 \mathrm{~nm}$ to $18 \mu \mathrm{m}$. The temporal pulsewidth was determined ( $68 \%$ confidence) to be $10 \pm 1 \mathrm{ps}(\mathrm{FWHM})$ at $10.6 \mu \mathrm{m}$ from pump probe measurements in InSb discussed later in this section. A beam waist $w_{0}$ of 120 to $140 \mu \mathrm{m}$ was determined from fitting the $\mathrm{InSb}$ $\mathrm{Z}$-scan results across the measured spectral range from 8 to $12 \mu \mathrm{m}$ at irradiances well below the $I_{c r}$. Examples of the Z-scan data and fitting are shown in Fig. 4. It is found that for the smallest energy picosecond Z-scans (with small changes in transmittance $\sim 5 \%$ ); the fitting for $2 \mathrm{PA}$ is almost independent of the FCA cross section used allowing for a good estimation of the beam waist and 2PA absorption coefficient. It is also found through fitting of data taken at larger irradiance levels for the FCA cross section that the values obtained are consistent with the theoretical values of $\sigma_{\mathrm{FCA}}$ over the entire temperature range studied. Therefore, theoretical values for FCA are adopted in modeling so that the only fitting parameter is 2PA. Part of the justification for doing this is that the theoretical values for FCA cross section were independently verified by linear absorption except at the lower temperatures. The 2PA coefficients are compared to theory and to values determined by femtosecond Z-scans showing agreement between the data and model using the values for the FCA cross sections given by Ref. 19 .

Since the photogenerated free-carrier population increases for longer pulsewidths, the associated refraction is also more 


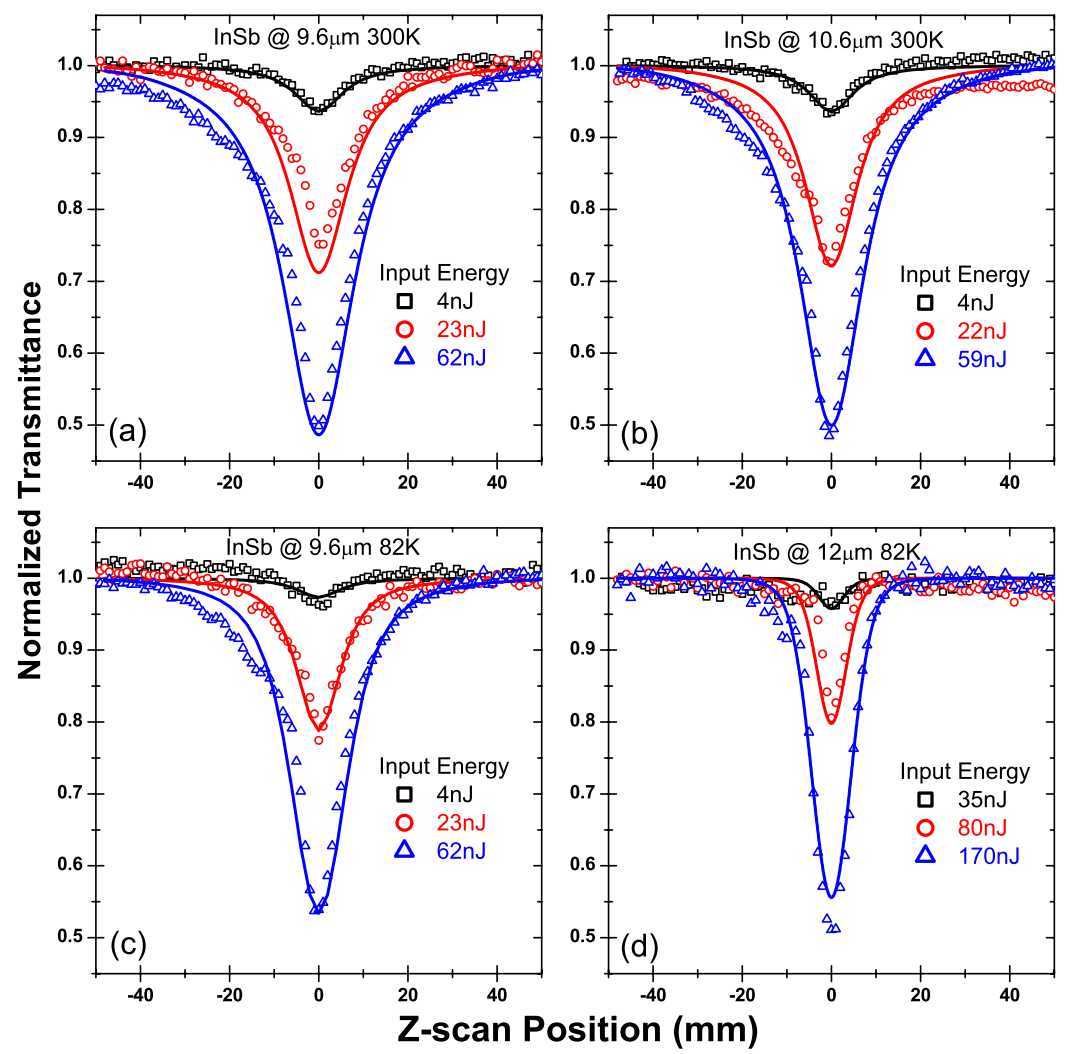

FIG. 4. (Color online) Open-aperture Z-scan data using $300 \mathrm{~K} \mathrm{InSb}$ at (a) $9.6 \mu \mathrm{m}$ fit with $\tau_{\mathrm{FWHM}}=10 \mathrm{ps}, w_{0}=140 \mu \mathrm{m}, \alpha_{2}=3.4 \mathrm{~cm} / \mathrm{MW}$, $\sigma_{\mathrm{FCA}}=7.3 \times 10^{-16} \mathrm{~cm}^{2}$, (b) $10.6 \mu \mathrm{m}$ fit with $\tau_{\mathrm{FWHM}}=10 \mathrm{ps}, w_{0}=140 \mu \mathrm{m}, \alpha_{2}=3.5 \mathrm{~cm} / \mathrm{MW}$, $\sigma_{\mathrm{FCA}}=6.8 \times 10^{-16} \mathrm{~cm}^{2}$, and $82 \mathrm{~K} \quad \mathrm{InSb}$ (c) $9.6 \mu \mathrm{m}$ fit with $\tau_{\mathrm{FWHM}}=10 \mathrm{ps}, w_{0}=140 \mu \mathrm{m}$, $\alpha_{2}=1.0 \mathrm{~cm} / \mathrm{MW}, \quad \sigma_{\mathrm{FCA}}=3.0 \times 10^{-15} \mathrm{~cm}^{2}, \quad(\mathrm{~d})$ $12 \mu \mathrm{m}$ fit with $\tau_{\mathrm{FWHM}}=10 \mathrm{ps}, w_{0}=140 \mu \mathrm{m}$, $\alpha_{3}=0.025 \mathrm{~cm}^{3} / \mathrm{MW}^{2}, \sigma_{\mathrm{FCA}}=3.4 \times 10^{-15} \mathrm{~cm}^{2}$. significant and potentially problematic. It is important to note that nonlinear refraction can result in collection losses that will incorrectly overestimate the value for nonlinear absorption. For our picosecond measurements we are confident that we are collecting all of the energy. The main sources of error are due to absolute energy calibration and pulsewidth measurement. Total experimental error for 2PA is therefore conservatively estimated to be $\pm 50 \%$. Although the absolute error in magnitude (due to noise and systematic error) is quite large, the relative error between spectral data is smaller (due to noise) allowing for accurate comparison of our data to the trend of the calculated spectra.

We also performed several frequency degenerate pumpprobe experiments at $10 \mu \mathrm{m}$ on the 0.54 -mm-thick intrinsic antireflective coated InSb sample. The probe beam was kept at a much smaller irradiance in order to not induce any nonlinear effects by itself and its transmittance was monitored for different delays with respect to the pump beam. The spot size ratio was approximately 4:1 (pump/probe) with the probe having an irradiance at least ten times smaller than that of the pump. We used a standard noncollinear geometry with an angle between the two beams of $\sim 7^{\circ}$. The polarization (linear) of the probe was rotated by $90^{\circ}$ with respect to the pump to minimize coherent artifacts which would have complicated the interpretation of the experimental data. Potential complications related to 2PA anisotropy are eliminated by using a $\langle 111\rangle$ crystal. These transient experiments provided a few advantages over the other single beam techniques. They allowed for the estimation of the employed pulsewidths directly while effectively decoupling the bound-electronic effects from the free-carrier effects. The short delay curves $(\sim 100 \mathrm{ps})$ shown in Fig. 5(a) allow for the monitoring of the free-carrier population buildup and for the determination of the 2PA coefficients. It should be noted here that in general, the measured 2PA coefficient depends on the relative polarization state of the two beams as well as the crystal orientation. ${ }^{25}$ In our case, since the two beams are perpendicularly polarized, we use different 2PA coefficients for fitting. $\alpha_{2, \mathrm{~s}}$ corresponds to the "self" 2PA experienced by the pump beam and determines the density of generated photocarriers. The other coefficient, $\alpha_{2, \mathrm{C}}$ (cross) describes the absorption experienced by the probe beam due to the presence of the pump. The data taken with longer delays shown in Fig. 5(b) ( $\sim 1 \mathrm{~ns})$ show relaxation on a picosecond time scale due to the Auger recombination, which is much faster than the direct nonradiative recombination time which is usually on the order of microseconds. ${ }^{19,26}$ We were able to obtain a consistent fitting for several pump energies using the same parameters (see Fig. 9) and a fixed value for FCA as extracted from the linear measurements. These experiments provide a much better indication for the magnitude of the Auger coefficient as the fits suggest a value of $C=6.5 \times 10^{-26} \mathrm{~cm}^{6} / \mathrm{s}$ with a relative error $(68 \%$ confidence $)$ of about $\pm 20 \%$. This is possible since at longer delays the only absorption present is due to the free-carrier population, and by monitoring the transmittance one can monitor directly the evolution of the carrier population. Previous measurements were done mostly employing much larger densities of photogenerated carriers through one photon pumping and yielded numbers spanning more than one order of magnitude. ${ }^{26}$ Our recent publication discussing nonlinear refraction and Auger recombination in InSb used a similar value to fit previously published data. ${ }^{14}$ 


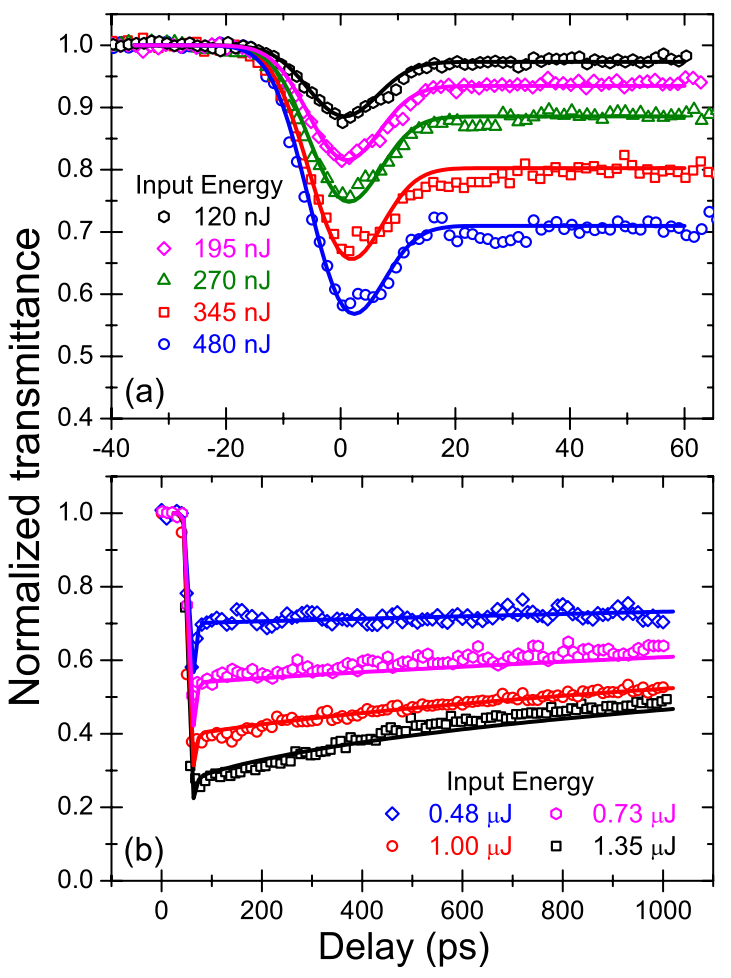

FIG. 5. (Color online) Degenerate pump-probe data in InSb at $10 \mu \mathrm{m}$ with cross-polarized beams; both the (a) short and (b) long delay curves are fit with the same parameters: $\tau_{\mathrm{FWHM}}=10 \mathrm{ps}$, $\sigma_{\mathrm{FCA}}=6.8 \times 10^{-16} \mathrm{~cm}^{-2}, \quad w_{0}=500 \mu \mathrm{m}, \quad L=450 \mu \mathrm{m}, \quad \alpha_{2, \mathrm{~S}}$ $=1.4 \mathrm{~cm} / \mathrm{MW}, \alpha_{2, \mathrm{C}}=1.9 \mathrm{~cm} / \mathrm{MW}, C=6.5 \times 10^{-26} \mathrm{~cm}^{6} / \mathrm{s}$. Linearly polarized beams are used in these experiments.

\section{Two-photon absorption}

The 2PA absorption values measured by Z-scan agree well with the spectral shape predicted by the detailed theory of Ref. 19 for both spectral and thermal variation as shown in Figs. 6 and 7. It is important to note that fitting the femtosecond data gives the lowest possible 2PA value as the error bars in Fig. 6 suggest, since the pulsewidths used in fitting are calculated assuming the pulsewidths at the output of the OPG are transform limited. Picosecond Z-scan data independently confirm the predicted spectral and temperature dependence of 2PA. Additionally, the results for femtosecond Z-scans and picosecond Z-scans at $300 \mathrm{~K}$ shown in Fig. 6 agree well with each other, $\alpha_{2} \sim 3 \mathrm{~cm} / \mathrm{MW}$. The absolute values also agree within a factor of 3 to the values calculated using the simple parabolic band model of 2PA with an empirical constant $\left(K_{2}=3.1 \mathrm{eV}^{5 / 2} \mathrm{~cm} / \mathrm{MW}\right)$ previously determined for semiconductors. ${ }^{9}$ The thermal variation in $2 \mathrm{PA}$ due to the change in band gap energy agrees slightly better with the predictions of Ref. 19 than with that of Ref. 6 and the difference in absolute magnitude between the theory of Ref. 19 and the data is within experimental error, except very near the band edge.

To compare all the data to theory of Ref. 6 simultaneously it is useful to scale the data in order to isolate the spectral or band gap dependence. The portion of Eq. (1) in the brackets is called $F_{2}$ or the spectral function. The scaled 2PA coefficient is obtained by dividing the measured 2PA value by $F_{2}$ and $\sqrt{E_{p}}$ then multiplying by $n^{2}$. A comparison of all the 2PA data with the scaling functions is shown in Fig. 8. As seen in Fig. 8, if the theory of Ref. 6 is scaled by setting $K_{2}=1.9 \mathrm{eV}^{5 / 2} \mathrm{~cm} / \mathrm{MW}$, the spectral dependencies closely match the predictions of Ref. 19. Thus the picosecond values agree with both theories if this value of $K_{2}$ is used. This level of agreement with Wherrett's theory, Ref. 6, using an empirical scaling constant demonstrates the utility of a simple twoparabolic band model as well as the accuracy of an extrapolated value based on the scaling rules derived from it.

\section{E. Three-photon absorption}

At wavelengths longer than the 2PA band edge, 3PA is the dominant absorption mechanism. This is the case for InSb at $80 \mathrm{~K}$ with wavelengths longer than $10.9 \mu \mathrm{m}$. In this work, 3PA in $\mathrm{InSb}$ at $80 \mathrm{~K}$ is determined by fitting picosecond Z-scan data shown in Fig. 4 to give $a_{3} \approx 0.025 \pm 0.012 \mathrm{~cm}^{3} / \mathrm{MW}^{2}$ at $12 \mu \mathrm{m}$. We recently calculated the degenerate 3PA spectrum of $\mathrm{ZnSe}$ using perturbation theory based on a Kane four-band model consisting of three valence bands (heavy-hole, light-hole, and split-off) and one conduction band. ${ }^{27}$ This model for zincblende structures accounts for the nonparabolicity of the bands and nonzone-center wave functions. Our experimental 3PA results for $\mathrm{ZnSe}$ matched the measured spectral shape, although the predicted values were a factor of 3.2 smaller than the experimental data for $\mathrm{ZnSe}$. Our theoretical value for InSb at $80 \mathrm{~K}$ using $E_{g}=0.228 \mathrm{eV}$ is $\alpha_{3} \approx 0.012 \mathrm{~cm}^{3} / \mathrm{MW}^{2}$
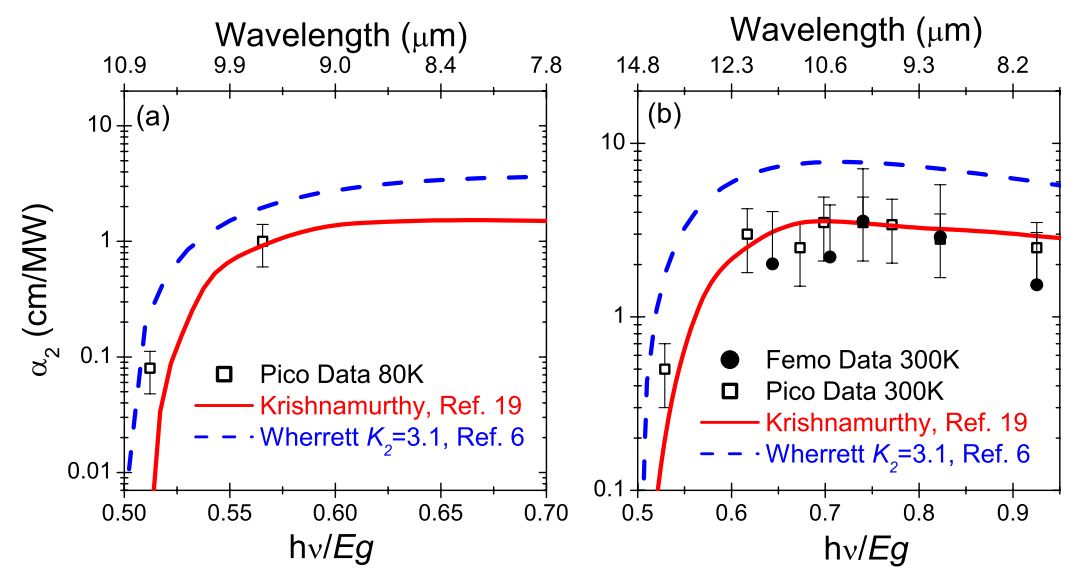

FIG. 6. (Color online) Comparison of 2PA values obtained from fitting femtosecond and picosecond Z-scan data with Wherrett's theory, Ref. 6, scaled by an empirical factor and more recent theory, Ref. 19 at (a) $80 \mathrm{~K}$ and (b) $300 \mathrm{~K}$. 

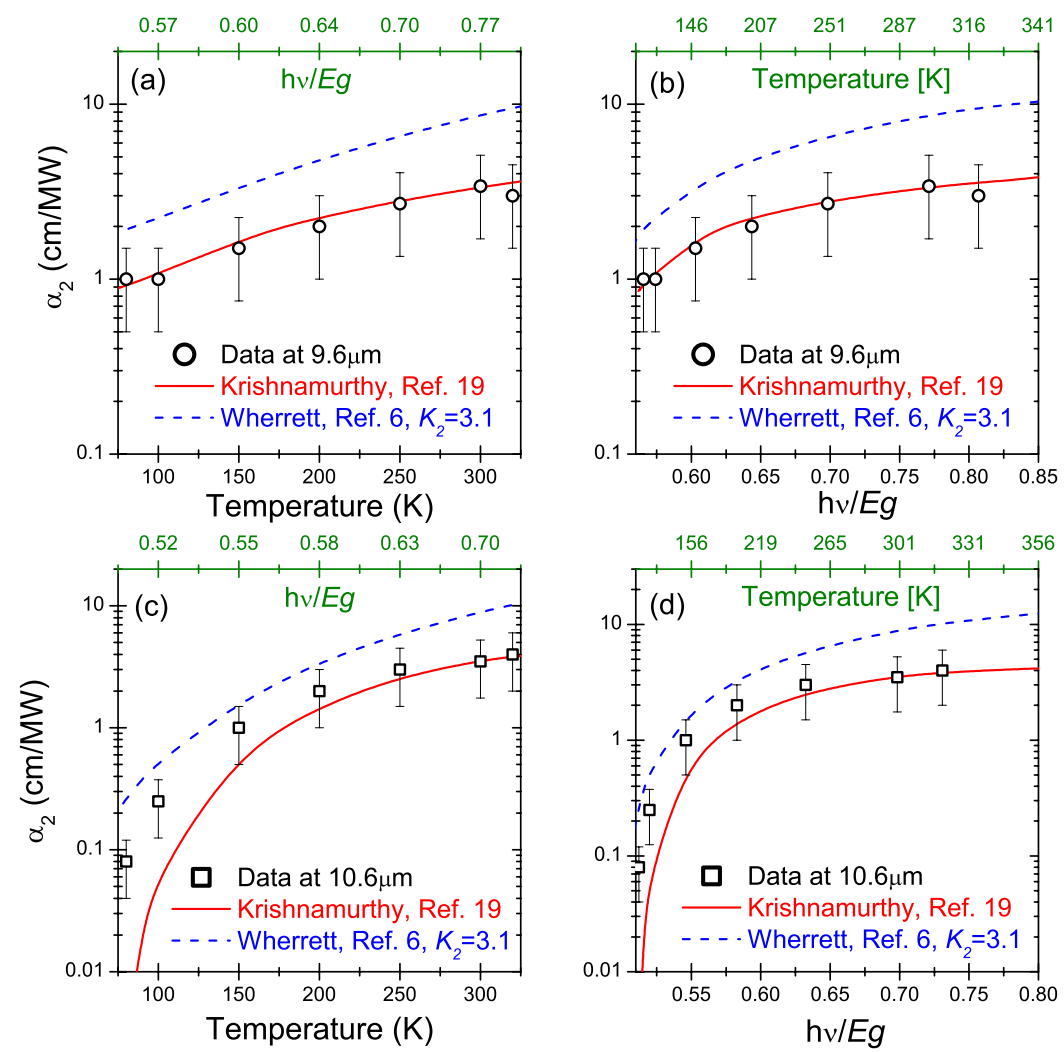

FIG. 7. (Color online) Picosecond Z-scan results for 2PA vs. temperature and vs photon energy normalized to the band gap vs temperature (a) and energy (b) at $9.6 \mu \mathrm{m}$ and vs temperature (c) and energy (d) at $10.6 \mu \mathrm{m}$ compared to theory. at $12 \mu \mathrm{m}$ is approximately a factor of 2 smaller than the experimental value. It should be noted here the agreement of this data with the 3PA model is achieved using the theoretical value for FCA at low temperature and a better fit for the largest energy used could be obtained by slightly varying the FCA value.

\section{F. Nanosecond experiments}

Nanosecond experiments are used to test our full model for nonlinear transmission, including the free-carrier recombination terms starting with irradiances showing linear transmittance up to the damage threshold. The sample is held in focus for two reasons: first, it is easier to determine the damage threshold by slowly increasing the energy; second, it proved difficult to scan the sample through focus to perform Z-scan measurements as it was mounted in a large CTICryogenics Model 22 helium cryostat which was used to enable measurements at temperatures down to $10 \mathrm{~K}$.

A single mode (both temporal and spatial) transverse excitation atmospheric pressure $\mathrm{CO}_{2}$ laser with a gain-switched pulsewidth of 150 ns FWHM and a beam waist $w_{0}$ of $180 \mu \mathrm{m}$ (measured by knife-edge scans) is used to obtain data from the linear to nonlinear regime to the laser-induced damage threshold (LIDT). The single longitudinal mode is obtained using a low pressure discharge cell within the laser cavity (Pulse Systems, model LP-15) low pressure, transverse excitation $\mathrm{CO}_{2}$ gas laser gain cell. The LIDT is reported $^{28,29}$ to be $1-2 \mathrm{~J} / \mathrm{cm}^{2}$ which is similar to the fluence levels that result in surface damage in this work. The temporal pulse profile is asymmetric [shown in the inset of Fig. $9(\mathrm{a})]$ and so it is important that the profile is recorded for modeling and monitored during the experiment. It is long enough to be measured using a digital storage oscilloscope (Tektronix, TDS680C, 1GHz/5Gs) with a fast gold-doped germanium detector. A typical profile and normalized nonlinear transmittance data recorded at $80 \mathrm{~K}$ and $300 \mathrm{~K}$ with the corresponding fittings are shown in Fig. 9.

The importance of taking the temperature dependence of the FCA cross section into account when modeling nonlinear absorption is demonstrated by the improved agreement obtained for 2PA at low temperature by using the FCA values predicted by theory in Fig. 10. Attempting to fit the nonlinear transmittance data with a fixed, temperature independent, FCA cross section of $\sigma_{\mathrm{FCA}}=8.6 \times 10^{-16} \mathrm{~cm}^{2}$ gives best fit values for $2 \mathrm{PA}$ which produce a spectrum inconsistent with the temperature dependence of 2PA as predicted by theory and verified by picosecond measurements. Using the temperature dependent values for FCA cross sections (with $\sigma_{\mathrm{FCA}}=3.5 \times 10^{-15} \mathrm{~cm}^{2}$ at $80 \mathrm{~K}$ and $\sigma_{\mathrm{FCA}}=6.8 \times 10^{-16} \mathrm{~cm}^{2}$ at $300 \mathrm{~K}$ ) allows for the accurate determination of $2 \mathrm{PA}$ as shown in Fig. 10. The nanosecond data is finally fit using FCA cross sections and 2PA coefficients consistent with theory $^{19}$ and obtained from FTIR measurements and picosecond Z-scan data. The data agrees remarkably well with the model using absorption values determined with shorter pulses and recombination rates that are consistent with the published ${ }^{26}$ range of values at room temperature $B \approx\{1-7\} \times 10^{-11} \mathrm{~cm}^{3} / \mathrm{s}$ and $C \approx\{4-2000\} \times 10^{-26} \mathrm{~cm}^{6} / \mathrm{s}$ and previous ${ }^{30}$ work at low temperatures $\left(B \approx 1 \times 10^{-9}\right)$.

\section{G. Exciton enhancement}

It has been suggested that exciton enhancement should be considered in the theoretical calculation of 2PA 

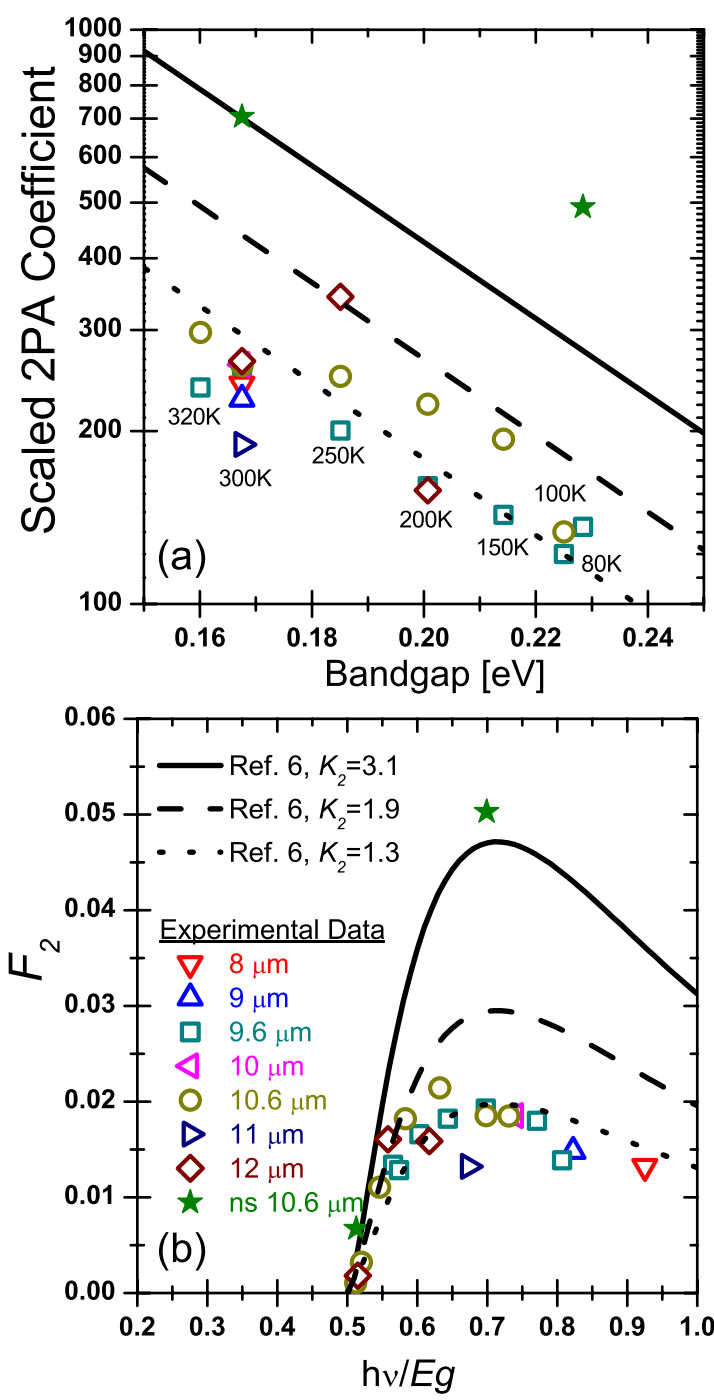

FIG. 8. (Color online) (a) 2PA coefficient scaled (by isolating the $E_{g}^{-3}$ in Eq. (1) from picosecond Z-scan experiments and nanosecond results at $300 \mathrm{~K}$ and $80 \mathrm{~K}$ compared to Wherrett's scaling theory from Ref. 6 where the solid line is generated using $K_{2}=3.1 \mathrm{eV}^{5 / 2} \mathrm{~cm} / \mathrm{MW}$, the dashed line a value of $K_{2}=1.9 \mathrm{eV}^{5 / 2} \mathrm{~cm} / \mathrm{MW}$, and the dotted line a value of $K_{2}=1.3 \mathrm{eV}^{5 / 2} \mathrm{~cm} / \mathrm{MW}$. (b) Same as for (a) except that here the data is scaled from Eq. (1) to isolate the $F_{2}$ spectral dependence. coefficients. ${ }^{31}$ Exciton enhancement is an increase in the absorption rate due to transitions that include energy levels of exciton states near the bottom of the conduction band. The discrepancy between theoretical and measured values observed in this work is larger near the band edge, which is consistent with the predicted spectral behavior of exciton enhancement. ${ }^{32}$ Therefore, a comparison of the picosecond data at $9.6 \mu \mathrm{m}$ and $10.6 \mu \mathrm{m}$ to both the theory of Ref. 6 without empirical factors and the theory of Ref. 19 while including exciton enhancement of 2PA is shown in Fig. 11. Nanosecond data at $10.6 \mu \mathrm{m}$ modeled with 2PA as a free parameter is included for the same comparison in Fig. 12. The picosecond data at $300 \mathrm{~K}$ agrees best with Krishnamurthy's theory while the nanosecond data agrees best with Wherrett's including exciton enhancement. Below $150 \mathrm{~K}$ the picosecond data agrees best with Krishnamurthy's theory with exciton enhancement and the nanosecond data agrees best with Wherrett's including exciton enhancement. In between 150 and $250 \mathrm{~K}$ the both sets of data agree best with Wherrett's theory without exciton enhancement. Overall, the two sets of data show good agreement with each other and consistently show improved agreement with theory at low temperature when exciton enhancement is included.

\section{H. Carrier recombination rates}

The Shockley-Read-Hall recombination involves energy levels related to crystal defects, and is therefore different from sample to sample. The SRH lifetime is taken to be a constant of $2 \mu \mathrm{s}$ and it is found that this process is negligible for fitting our data and values ranging from 2 to $0.5 \mu \mathrm{s}$ make little difference in the fitting. The parameter $B$ is expected to decrease with increasing temperature. ${ }^{27}$ Radiative recombination is therefore varied when fitting the nanosecond nonlinear transmittance data from $B \approx 0.7 \times 10^{-10} \mathrm{~cm}^{3} / \mathrm{s}$ at $300 \mathrm{~K}$ to $B \approx 3.3 \times 10^{-10} \mathrm{~cm}^{3} / \mathrm{s}$ at $80 \mathrm{~K}$. Using larger values for the radiative recombination rate results in smaller fitted values for the Auger recombination rate. Including radiative recombination also results in a better overall fit at low temperature. The Auger rates were determined from picosecond pump-probe experiments at room temperature to be $C=6.5 \times 10^{-26} \mathrm{~cm}^{6} / \mathrm{s}$ and from fitting the nanosecond nonlinear transmittance data to be $C$ $\approx 7 \times 10^{-26} \mathrm{~cm}^{6} / \mathrm{s}$ at $300 \mathrm{~K}$ and $C \approx 3 \times 10^{-25} \mathrm{~cm}^{6} / \mathrm{s}$ at 80
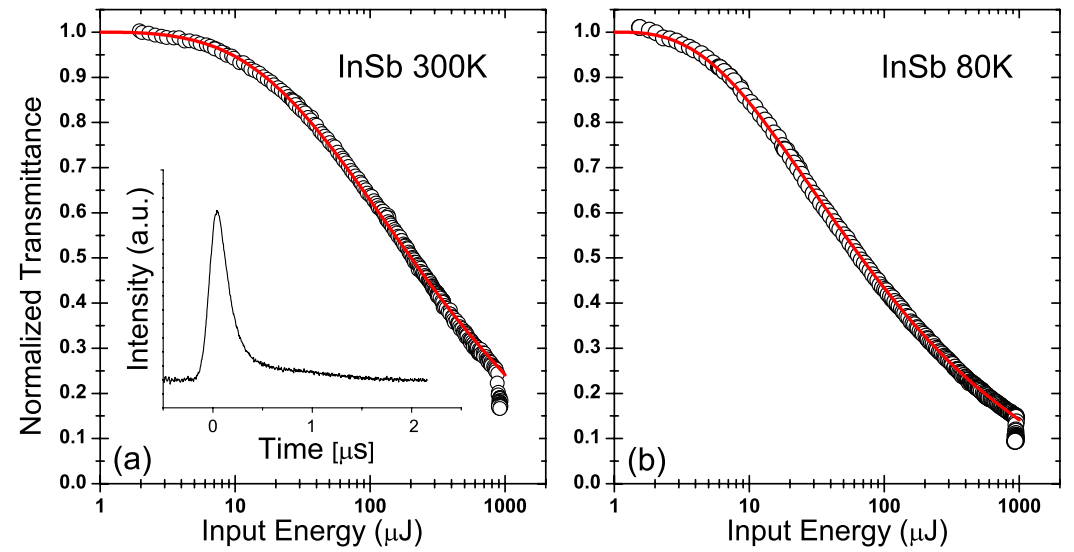

FIG. 9. (Color online) Nonlinear transmission data up to the damage threshold at $\lambda=10.6 \mu \mathrm{m}$ of InSb (a) $300 \mathrm{~K}$ fit with $w_{0}=180 \mu \mathrm{m}, \sigma_{\mathrm{FCA}}=6.75$ $\times 10^{-16} \mathrm{~cm}^{2}, \tau=2 \mu \mathrm{s}, B=0.7 \times 10^{-10} \mathrm{~cm}^{3} / \mathrm{s}, \alpha_{2}$ $=9.5 \mathrm{~cm} / \mathrm{MW}, C=7 \times 10^{-26} \mathrm{~cm}^{6} / \mathrm{s}$ and (b) $80 \mathrm{~K}$ fit with $w_{0}=180 \mu \mathrm{m}, \sigma_{\mathrm{FCA}}=32.4 \times 10^{-16} \mathrm{~cm}^{2}, \tau$ $=2 \mu \mathrm{s}, B=3.3 \times 10^{-10} \mathrm{~cm}^{3} / \mathrm{s}, \quad \alpha_{2}=0.5 \mathrm{~cm} / \mathrm{MW}$, $C=30 \times 10^{-26} \mathrm{~cm}^{6} / \mathrm{s}$. The drop in transmission near $1 \mathrm{~mJ}$ indicates the onset of damage. Inset shows the input pulse from the nanosecond $\mathrm{CO}_{2}$ laser which is included in the modeling. 


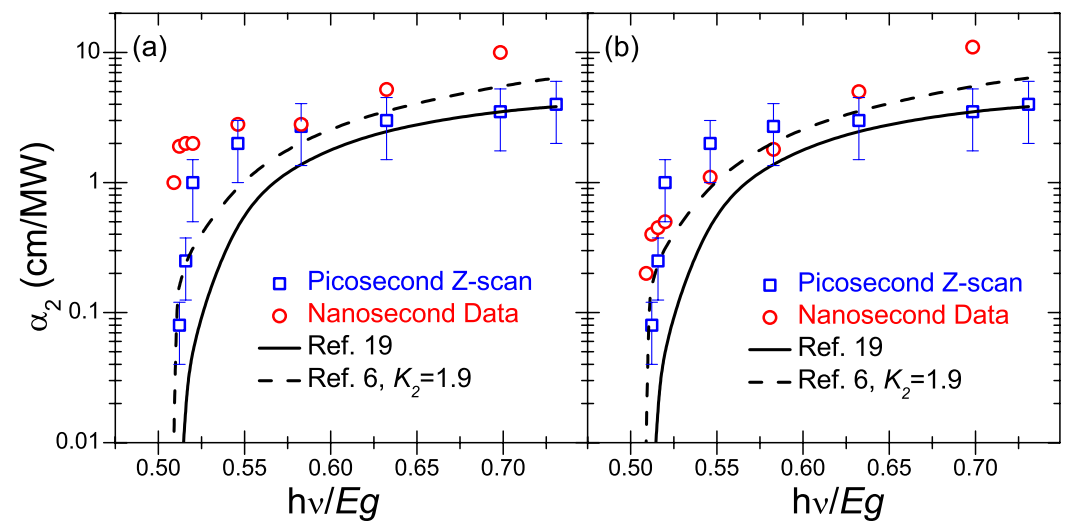

FIG. 10. (Color online) Comparison of values obtained from fitting nanosecond data for 2PA (a) with a constant FCA cross section and (b) with a temperature-dependent FCA cross section.
$\mathrm{K}$. This trend is slightly contradictory to the expected trend since the band gap energy is considerably larger at lower temperature. Auger rates are generally smaller for larger band gap semiconductors, ${ }^{26}$ and it is predicted by Ref. 19 that the Auger coefficient should be approximately four times smaller at $80 \mathrm{~K}$ than $300 \mathrm{~K}$. The room-temperature value for Auger recombination that we measure with nanosecond experiments is at the lower end but within the range of reported values. ${ }^{26}$ Auger recombination is the dominant decay term for modeling the nanosecond data, and although literature values for SRH and radiative decay were included

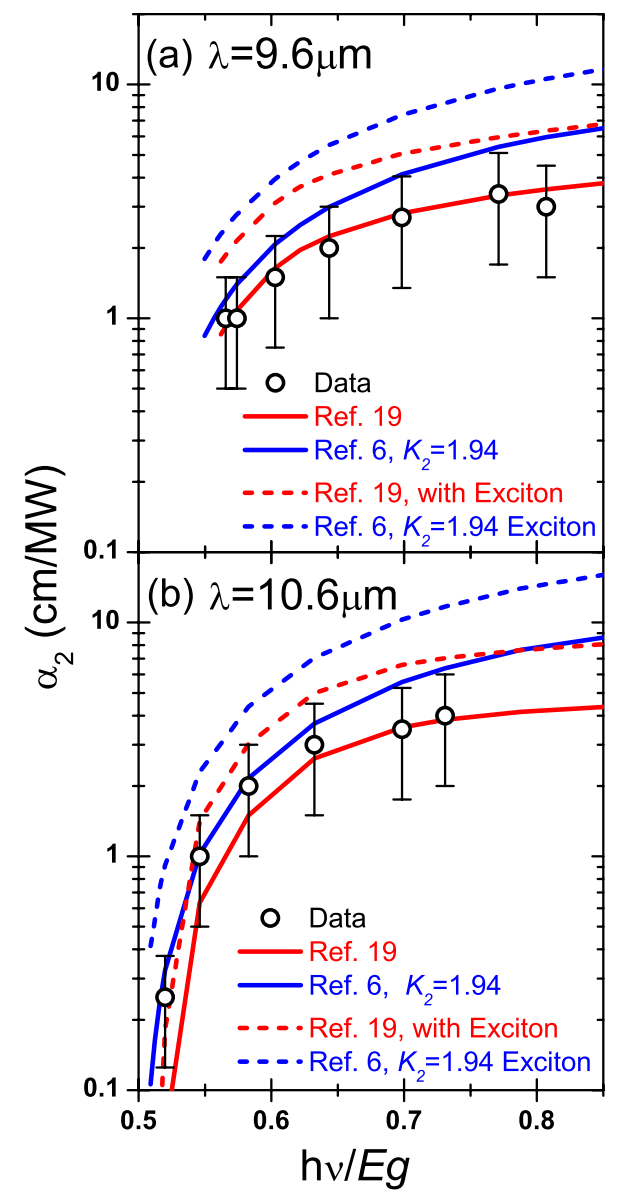

FIG. 11. (Color online) 2PA picosecond data vs temperature controlled band gap at (a) $9.6 \mu \mathrm{m}$ and (b) $10.6 \mu \mathrm{m}$ with and without exciton enhancement. in the model for nanosecond data they have negligible effects for the fitting of picosecond data.

\section{CONCLUSIONS}

In this paper we present an investigation of both the temperature and spectral behavior of 2PA and FCA in InSb using tunable infrared sources with pulsewidths spanning six orders of magnitude from $\sim 160$ fs to $\sim 150$ ns which are required to more accurately determine the photophysical parameters and their nature. We compare our experimental results to predictions from a simple two-parabolic band model $^{6}$ and to more recent calculations that include a more accurate band structure. ${ }^{19}$ A methodology is demonstrated using different temperatures, pulsewidths and photon energies to measure nonlinear absorption and allowing separate as well as combined modeling of multiphoton absorption and FCA parameters.

The measured 2PA spectrum agrees well with the spectral shape predicted by both theories for thermal variations (from 80 to $300 \mathrm{~K}$ ) at fixed wavelengths and for spectral variations (from 8 to $12 \mu \mathrm{m}$ ) at fixed temperatures. ${ }^{6,19}$ The measured

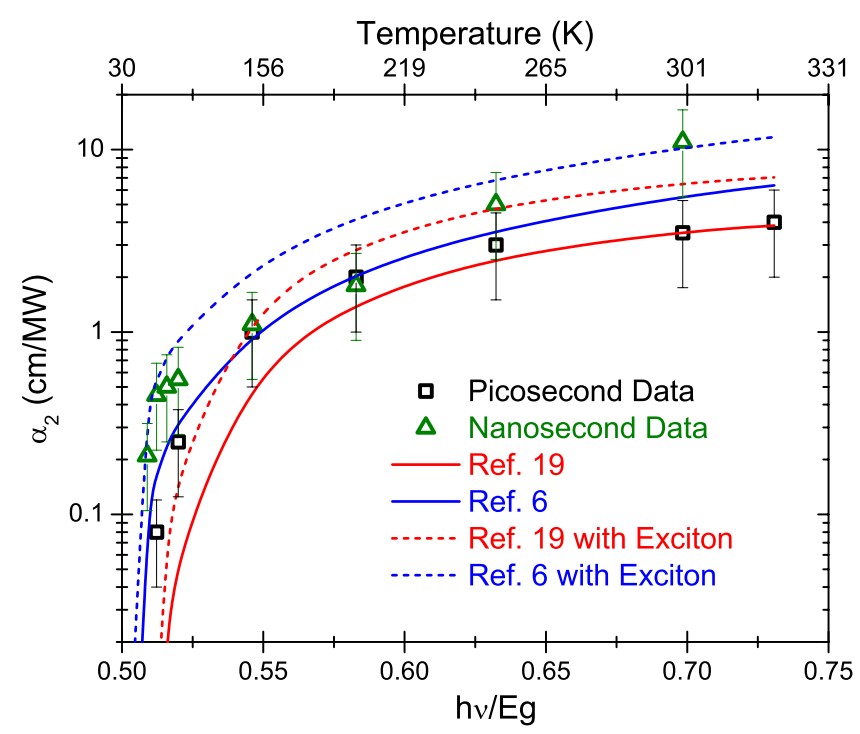

FIG. 12. (Color online) Picosecond and nanosecond 2PA data at $10.6 \mu \mathrm{m}$ compared to theory with and without exciton enhancement. 
values agree with the new theoretical values for 2PA (Ref. 19) and $^{27}$ 3PA within error; however, the discrepancy between data and theory is larger near the absorption edge consistent with exciton enhancement. A comparison of both theories with the picosecond data including exciton enhancement shows improved agreement near the absorption edge. We note that the enhancement of absorption near the edge indicates that there are states that become resonant near the band edge which are not included in the model, and these states are not necessarily only excitonic states.

FCA cross section values are confirmed through modeling of 1PA, 2PA, and 3PA data. In order to obtain a consistent analysis of the 2PA spectra for nanosecond pulsewidths, it is necessary to consider that the FCA cross section is temperature dependent. FCA cross section values were measured at and above $200 \mathrm{~K}$ through 1PA via temperature controlled Fourier transform infrared measurements and temperaturecontrolled linear transmittance measurements of picosecond pulses. This along with FCA values used for the entire temperature range from 80 to $300 \mathrm{~K}$ to fit picosecond Z-scans and nanosecond nonlinear transmittance measurements, con- firm that the FCA cross section is temperature dependent as recently predicted by theory. ${ }^{19}$

Recombination rates are included for modeling nanosecond data, and it is found through modeling this data that these rates change with sample temperature. The agreement found at room temperature is quite good; however, the trend found with varying temperature is slightly contradictory with the expected trend for the Auger process. Since the band gap energy is considerably larger at lower temperatures the Auger rates are generally smaller for larger band gap semiconductors and it is predicted by Ref. 19 that the Auger coefficient should be approximately four times smaller at $80 \mathrm{~K}$ than $300 \mathrm{~K}$.

\section{ACKNOWLEDGMENTS}

This work is supported by the Air Force Office of Scientific Research (AFOSR) under Grant No. FA95500410200 and the National Science Foundation under Grant No. ECS 0524533 .
*Corresponding author; ewvs@creol.ucf.edu

${ }^{1}$ M. Göppert-Mayer, Ann. Phys. 401, 273 (1931).

${ }^{2}$ W. Kaiser and C. G. B. Garrett, Phys. Rev. Lett. 7, 229 (1961).

${ }^{3}$ L. V. Keldysh, Sov. Phys. JETP 21, 1135 (1965).

${ }^{4}$ H. D. Jones and H. R. Reiss, Phys. Rev. B 16, 2466 (1977).

${ }^{5}$ H. S. Brandi and C. B. de Araujo, J. Phys. C 16, 5929 (1983).

${ }^{6}$ B. S. Wherrett, J. Opt. Soc. Am. B 1, 67 (1984).

${ }^{7}$ C. R. Pidgeon, B. S. Wherrett, A. Johnston, J. Dempsey, and A. Miller, Phys. Rev. Lett. 42, 1785 (1979).

${ }^{8}$ D. C. Hutchings and E. W. Van Stryland, J. Opt. Soc. Am. B 9, 2065 (1992).

${ }^{9}$ E. O. Kane, J. Phys. Chem. Solids 1, 249 (1957).

${ }^{10}$ M. Sheik-Bahae, D. J. Hagan, and E. W. Van Stryland, Phys. Rev. Lett. 65, 96 (1990).

${ }^{11}$ E. W. Van Stryland, M. A. Woodall, H. Vanherzeele, and M. J. Soileau, Opt. Lett. 10, 490 (1985).

${ }^{12}$ M. Sheik-Bahae, D. J. Hagan, and E. W. Van Stryland, IEEE J. Quantum Electron. 27, 1296 (1991).

${ }^{13}$ M. Sheik-bahaei, P. Mukherjee, and H. S. Kwok, J. Opt. Soc. Am. B 3, 379 (1986).

${ }^{14}$ V. Dubikovskiy, D. J. Hagan, and E. W. Van Stryland, J. Opt. Soc. Am. B 25, 223 (2008).

${ }^{15}$ M. Sheik-Bahae, A. A. Said, T.-H. Wei, D. J. Hagan, and E. W. Van Stryland, IEEE J. Quantum Electron. 26, 760 (1990).

${ }^{16}$ M. Sheik-bahae, A. A. Said, and E. W. Van Stryland, Opt. Lett. 14, 955 (1989).

${ }^{17}$ C. L. Littler and D. G. Seiler, Appl. Phys. Lett. 46, 986 (1985).

${ }^{18}$ S. W. Kurnick and J. M. Powell, Phys. Rev. 116, 597 (1959).
${ }^{19}$ S. Krishnamurthy, Z. G. Yu, L. P. Gonzalez, and S. Guha, J. Appl. Phys. 101, 113104 (2007).

${ }^{20}$ M. C. Hoffmann, J. Hebling, H. Y. Hwang, K. L. Yeh, and K. A. Nelson, Phys. Rev. B 79, 161201(R) (2009).

${ }^{21}$ N. A. van Dantzig and P. C. M. Planken, Phys. Rev. B 59, 1586 (1999).

${ }^{22}$ M. C. Hoffmann and D. Turchinovich, Appl. Phys. Lett. 96, 151110 (2010).

${ }^{23}$ M. P. Hasselbeck, E. W. Van Stryland, and M. Sheik-Bahae, Phys. Rev. B 56, 7395 (1997).

${ }^{24}$ J. H. Bechtel and W. L. Smith, Phys. Rev. B 13, 3515 (1976).

${ }^{25}$ R. De Salvo, M. Sheik-Bahae, A. A. Said, D. J. Hagan, and E. W. Van Stryland, Opt. Lett. 18, 194 (1993).

${ }^{26}$ J. Piprek, Semiconductor Optoelectronic Devices: Introduction to Physics and Simulation (Academic, New York, 2003), Vol. 67.

${ }^{27}$ C. M. Cirloganu, P. D. Olszak, L. A. Padilha, S. Webster, D. J. Hagan, and E. W. Van Stryland, Opt. Lett. 33, 2626 (2008).

${ }^{28}$ A. Garg, K. N. Tripathi, A. Kapoor, and S. J. Bansal, Proc. SPIE 5629, 361 (2005).

${ }^{29}$ V. Dubikovskiy, Optical Limiting Numerical Modeling and Experiment, Department of Physics, University of Central Florida, Orlando, 2003.

${ }^{30}$ H. J. Fossum and B. Ancker-Johnson, Phys. Rev. B 8, 2850 (1973).

${ }^{31}$ C. C. Lee and H. Y. Fan, Phys. Rev. B 9, 3502 (1974).

${ }^{32}$ M. H. Weiler, Solid State Commun. 39, 937 (1981). 